\title{
Mechanism and Computer Simulation of a New Robot Hand for Potential Use as an Artificial Hand
}

\author{
Li-Ren Lin and Han-Pang Huang \\ Robotics Laboratory, Department of Mechanical Engineering. National Taiwan University, Taipei, Taiwan, Republic of China
}

\begin{abstract}
A prosthetic hand is essential to provide rehabilitation for individuals who lose a hand. A prosthetic hand serves two purposes: cosmetic and functional. In this paper, a prototype of the artificial hand with an emphasis on the functionality purpose is presented. A new mechanism, the NTU-Hand (NTU-Hand, patent number 107115, Taiwan, R.O.C.), which has 5 fingers with 17 degrees of freedom, has been designed and fabricated in our laboratory. Due to the special design of the mechanism, the hand has an uncoupled configuration in which each finger and joint are all individually driven. The size
\end{abstract}

of the hand is almost the same as a human hand. All actuators, mechanical parts, and sensors are on the hand. The compact design makes it feasible to adapt the hand to the injured wrist. A computer simulation with three-dimensional graphics was also built to evaluate the manipulable range of the artificial hand. From the results of this simulation, the relationship between the hand and the grasped object in a specific viewpoint can be obtained. Key Words: NTU-Hand-Prosthetic hand-Artificial hand-Hand mechanism design.
The prosthetic hand in the rehabilitation application usually performs a simple grip and serves a cosmetic function. An artificial hand for fine motion and dexterous manipulations is a challenging topic in the research and applications of both prosthetics and robotics. To be considered dexterous, a multifingered hand is required to act as a multipurpose gripping device for various tasks. Because most multifingered hands are designed to replace some of the work of human hands, they duplicate the shape and function of human hands. In addition to dexterous manipulation, the ability to perform stable grasp is also required. The size of the hand plays a significant part in the research. A sufficiently compact multifingered hand can be attached to the wrist of the injured hand or to the end of an industrial robot arm.

A dexterous prosthetic hand requires each finger and joint be individually driven, as in the human hand. Many multifingered hands have been developed that aim to achieve the dexterous requirements $(1-4)$. The number of fingers ranges from 3 to 5 . An example of a 3-fingered artificial hand is the JPL/Stanford hand. Each finger of

Received January 1996; revised June 1996.

Address correspondence and reprint requests to Prof. Han-Pang Huang, Robotics Laboratory, Department of Mechanical Engineering. National Taiwan Liniversity, Taipei, Taiwan 10674, R.O.C.

Presented in part at the Xth World Congress of the International Society for Artificial Organs, held November 14-18, 1995. Taipei. Taiwan, R.O.C. that hand has 3 degrees of freedom (DOFs) and is driven by 4 motors through tendon cables. Two parallel axis joints provide rotation, and the third proximal joint, perpendicular to the other joints, provides the sideward motion. Due to the large number of motors and strong coupling in the tendon configuration, the control system of the JPL/Stanford hand is very complicated.

The Utah/MIT hand (1) has 1 thumb and 3 fingers. Each finger of the hand has 4 joints. Three parallel joints provide the rotation, and the proximal joint supplies the lateral action. Due to the 4 DOFs in each finger, 8 independent tendons and pneumatic cylinders are required. Each pair of tendons must be kept tense to maintain the joint of the finger. Once a lateral motion is performed, 1 joint is activated, and the other 3 joints keep stationary so that tension on 8 tendons can be recalculated. The coupling problem also causes the Utah/MIT hand to use large number of actuators and complex control system (6). These 2 artificial hands are bulky because of their tendon driven configurations and associated control systems. As a result, they are restricted to use in robotics rather than prosthetics.

The Belgrade/USC hand ( 3 ) has 5 fingers and 4 motors: 2 motors for the thumb and 2 for the other fingers. Each finger has 3 parallel axis joints with an adaptability feature and 1 DOF. The hand provides only simple grasping capacity and not dexterous manipulation. The 


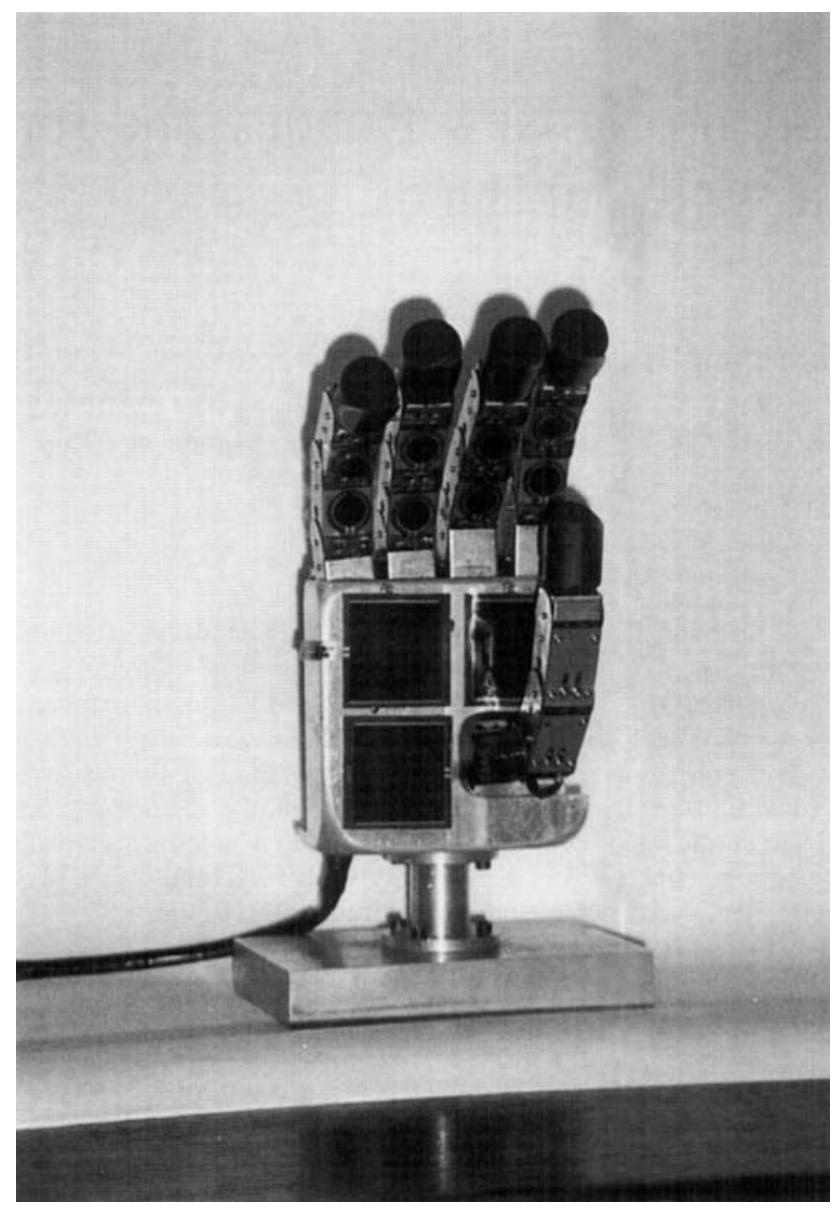

FIG. 1. The NTU-Hand is shown.

multifingered hand devised by the Yaskawa Electric Corporation has 3 fingers and 9 DOFs (4). This hand utilizes only its fingertips because of its bulky cylinder finger segments. These 2 hands are also not suitable candidates for prosthetics.

This paper explains the design and implementation of a dexterous artificial hand with potential use as a prosthetic. It is organized in two parts. The first part is the mechanical design of the NTU-Hand, as shown in Fig. 1. This part presents the design concept of the NTU-Hand and shows the detailed scheme of the mechanism. The specification and some issues of the mechanical considerations are also stated. The second part is the computer simulation. It is built to evaluate the manipulable range of the artificial hand with three-dimensional graphics. From the results of the simulation, the posture of the artificial hand an: the relationship between the hand and the grasped object in a specific viewpoint can be obtained. The control and grasping analysis of the NTUHand (7) are not addressed in this paper.

\section{HAND MECHANISM DESIGN}

The design of tendon driven cables is used in many artificial hands. One reason for this design is that the cables act as the muscles of the human hand. Another reason is that the actuators, reduction gears, and sensors can be remotely installed to keep the size of the hand small. Such a design means that the hand cannot be used in prosthetic applications when the numbers of joints and fingers are increased. In this paper, a new multifingered hand with an uncoupled driven mechanism and modular design was developed to limit the hand size so that the hand can be useful for both prosthetic and industrial applications.

The goals of the mechanism design are severalfold. The first addresses the functionality purpose, the number of fingers and DOFs. It is known that 3 fingers are required to grasp an object for a realistic model of the fingertips (8). However, the number of fingers and of DOFs of the artificial hand ideally should match those of the human hand. The second goal is the size. The size of the NTU-Hand, including the overall driver mechanism, is the same as that of the human hand. Limiting the size makes the hand suitable for rehabilitation purposes. Once all of the parts are packed in the hand itself, the hand can be easily attached to the wrist of the injured arm or to the industrial robot.

The third goal focuses on fabrication and maintenance. If the same parts are repeatedly used in the modular design of the hand, the types of parts will be reduced and the cost will decrease. Once the fingers are independent

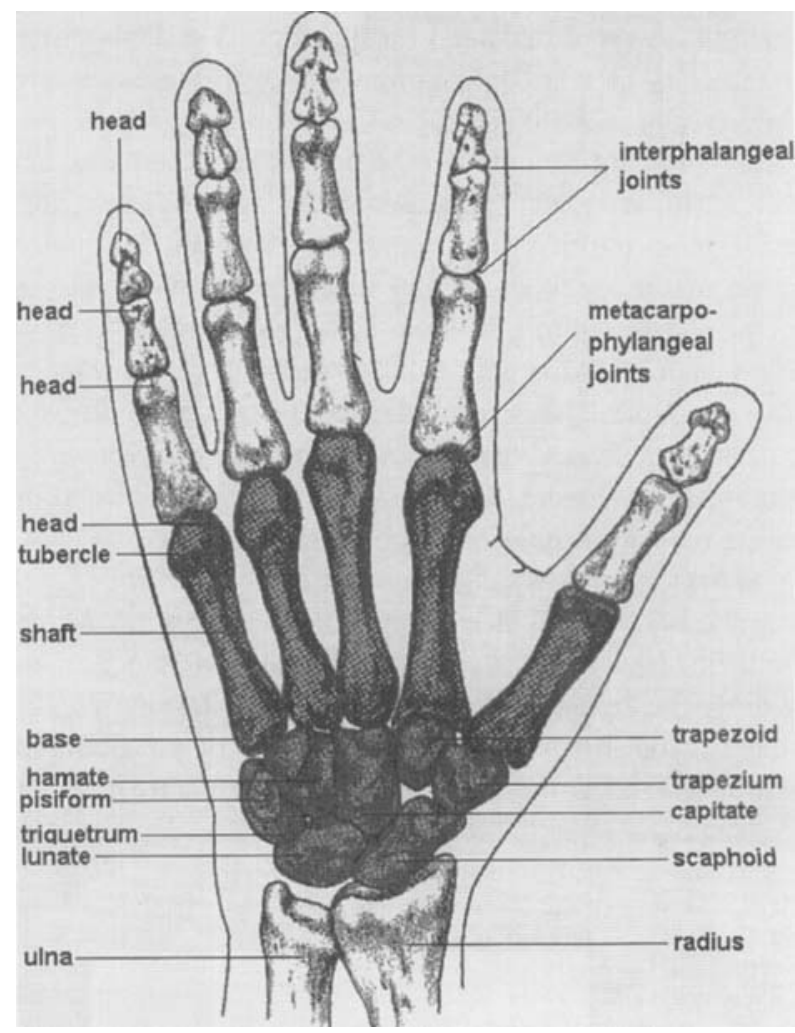

FIG. 2. The human hand (9) is shown. 


\begin{tabular}{|l|l|}
\hline 1. Finger tip & 6. Cover of the finger segment \\
\hline 2. Base of the finger tip & 7. High performance micro motor \\
\hline 3. Shaft & 8. Gear on the shaft of motor \\
\hline 4. Shell of the finger segment & 9. Seat of the motor \\
\hline 5A. Gear train A $(96: 1)$ & 10. Position sensor \\
\hline 5B. Gear train B $(812: 1)$ & 11. Tactile sensor \\
\hline
\end{tabular}

FIG. 3. The assembly of the fundamental finger is shown.

and interchangeable, maintenance simply requires replacing the damaged finger assembly without recalibrating the whole system. The fourth and final goal deals with the potential of improvement. The performance can be enhanced by replacing the parts whenever better materials are available. The main design, however, is preserved. Once the materials of transmission or the powers of the actuator are improved, the performance of the hand is also enhanced. For different applications, only the kind of material, and not the main design, is changed; e.g., the composite material for rehabilitation and the steel material for robotics applications.

According to the above goals, the design of the NTUHand was established. The NTU-Hand has 5 fingers with 17 DOFs. Both the thumb and the first finger have 4 joints: 2 at the knuckle; 1 between the proximal and middle finger segments; and 1 between the middle and distal finger segments. Other fingers have 3 joints but only 1 at the knuckle. The palm and each finger are equipped with tactile sensors to detect grasping force. The concept and details of design in the subsequent sections are shown.

\section{Hand mechanism}

The idea behind the design is obtained from the human hand. Because the structures of the fingers of the human hand are almost the same and independent, as shown in Fig. 2, this feature gave us the idea to design the hand beginning with the finger and to simplify the development. The human finger consists of several finger segments. This fact also inspired us to design independently driven finger segments for a complete finger. The auxiliary devices for the artificial finger are also required for the lateral motion, such as the function of the muscles in the palm.

\section{The finger mechanism}

The finger of the NTU-Hand consists of distal, middle, proximal, and base finger segments, as does the human finger. To ease the manufacture and assembly, group technology is applied to the design of the fingers and finger segments. The design should avoid the coupling problem of a tendon-driven structure and limit the size to make it useful for applications. Once an individual finger is constructed, the mechanism of the whole artificial hand is almost complete. To meet the requirement of the independent driver, it is essential to design a finger with all of the equipped parts.

The design of the finger is shown in Fig. 3. Each finger segment, except the distal segment, contains one highperformance micromotor that drives a set of specially arranged gear trains to rotate the previous finger segment, as shown in Fig. 4. The gear ratio of the middle and proximal finger segments is approximately 100, but the gear ratio of the base segment is approximately 1,000 for the sake of heavy load. The position sensor of each joint is also installed in each finger segment. It is driven 


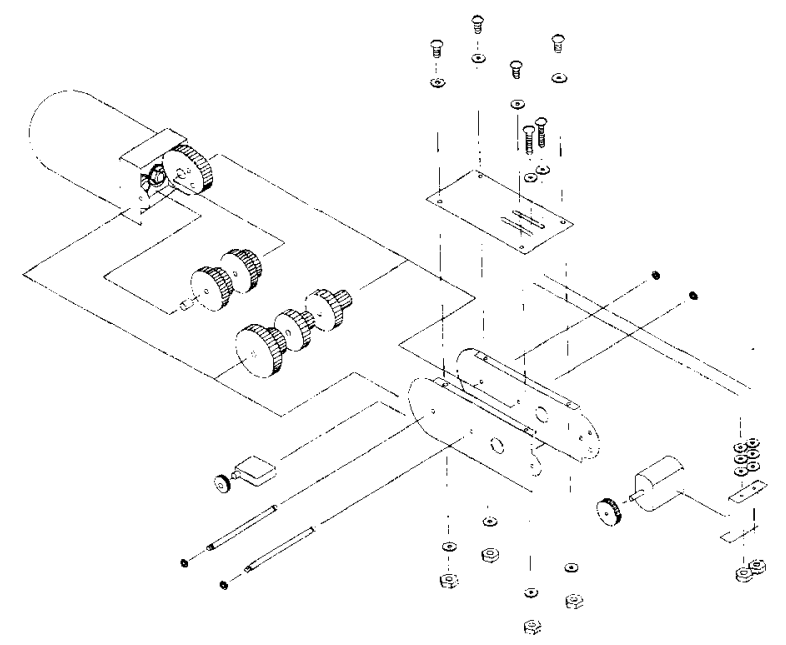

a

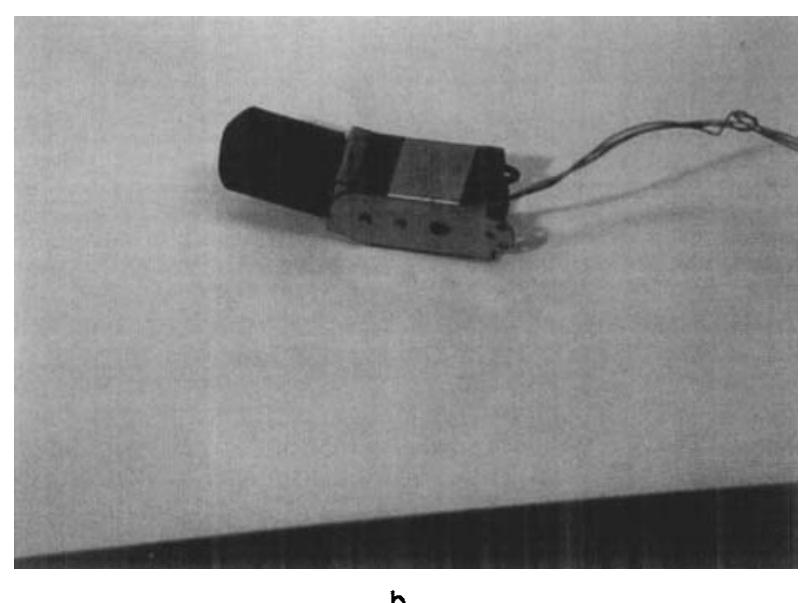

FIG. 4. Assembly of the distal segment and middle segment is shown in the assembly diagram and photograph. (A set of gear train, micromotor, and a potentiometer for the detection of joint variable are all packed in the middle segment (Fig. 3).

by the gear within the gear trains and is proportional to the angle of the finger joint.

There are many electrical wires in the inner space of each segment shell, and therefore the placement of sensors and motors must be taken into account during the assembly process. Because the position sensor is driven by a gear within the gear trains, its calibration must be accomplished during the assembly process.

\section{The auxiliary device for the lateral joints}

To ease the manufacturing effort, the lateral rotation of the thumb and the first finger is achieved by adding an auxiliary device to a fundamental finger, and the mechanical power is provided by an additional finger segment. The overall design scheme of the NTU-Hand is shown in Fig. 5. In the design of the lateral joint of the thumb, a large gear is attached to the thumb, and a modified finger segment is used to provide transmission. In the design of the lateral joint of the first finger, an adjustable linkage with a ball joint is used to transfer the mechanical power, the rotation of the gear is converted into a linear motion. The thumb and the first finger, each with 4 DOFs, provide approximately anthropomorphic motions similar to those of the human hand.

\section{The palm and the wrist}

The palm serves as a structural mounting base for the thumb, fingers, and the wrist. The last 3 fingers are fixed on the palm by a board that arranges the fingers to cooperate with the thumb and the first finger. The upper space of the palm provides the location to install the controller and various electronic components (10). The wrist shown in Fig. 5 is designed to connect the NTUHand to the end of the robot arm. Once the design of the NTU-Hand is modified for rehabilitation, the wrist needs to be changed to adapt to the injury.

\section{The tactile sensors}

One of the important sensing functions of the human hand is tactility. An artificial hand needs to be equipped with tactile sensors to detect contact force. The palm and each finger of the NTU-Hand are equipped with tactile sensors to detect grasping force. Due to cost issues and practical implementation, the tactile sensors are attached to the inner sides of finger segments and the palm shown in Figs. 1 and 6. The tactile sensor on the NTU-Hand is called a force-sensing resistor (FSR) (9). It acts as a variable resistor. Its specifications are shown in Table 1. Because the characteristics of FSR are nonlinear, as shown in Fig. 7, the FSR cannot be used directly. It must be processed to transfer into the linear scale by a digital processor. This value is then used for sensor integration.

\section{Satisfaction of the goals}

As mentioned before, there are at least four goals to be qualified and are discussed in this section.

\section{The functionality purpose}

The NTU-Hand has 5 fingers with 17 DOFs. All fingers have 3 parallel joints. The thumb and the first finger have additional joints to perform lateral motion. All other existing hands $(1,4,5)$ use at least 3 dexterous fingers to grasp whereas the NTU-Hand uses the middle finger to cooperate with the dexterous thumb and the first finger. This design makes grasping simple and achieves the highest degree of manipulability. The last 2 fingers of the NTU-Hand are used to improve the stability of object holding.

All 5 fingers of the NTU-Hand have a functional duty. From a cosmetic point of view, the 5 finger design resembles a human hand. In addition, the NTU-Hand, with 


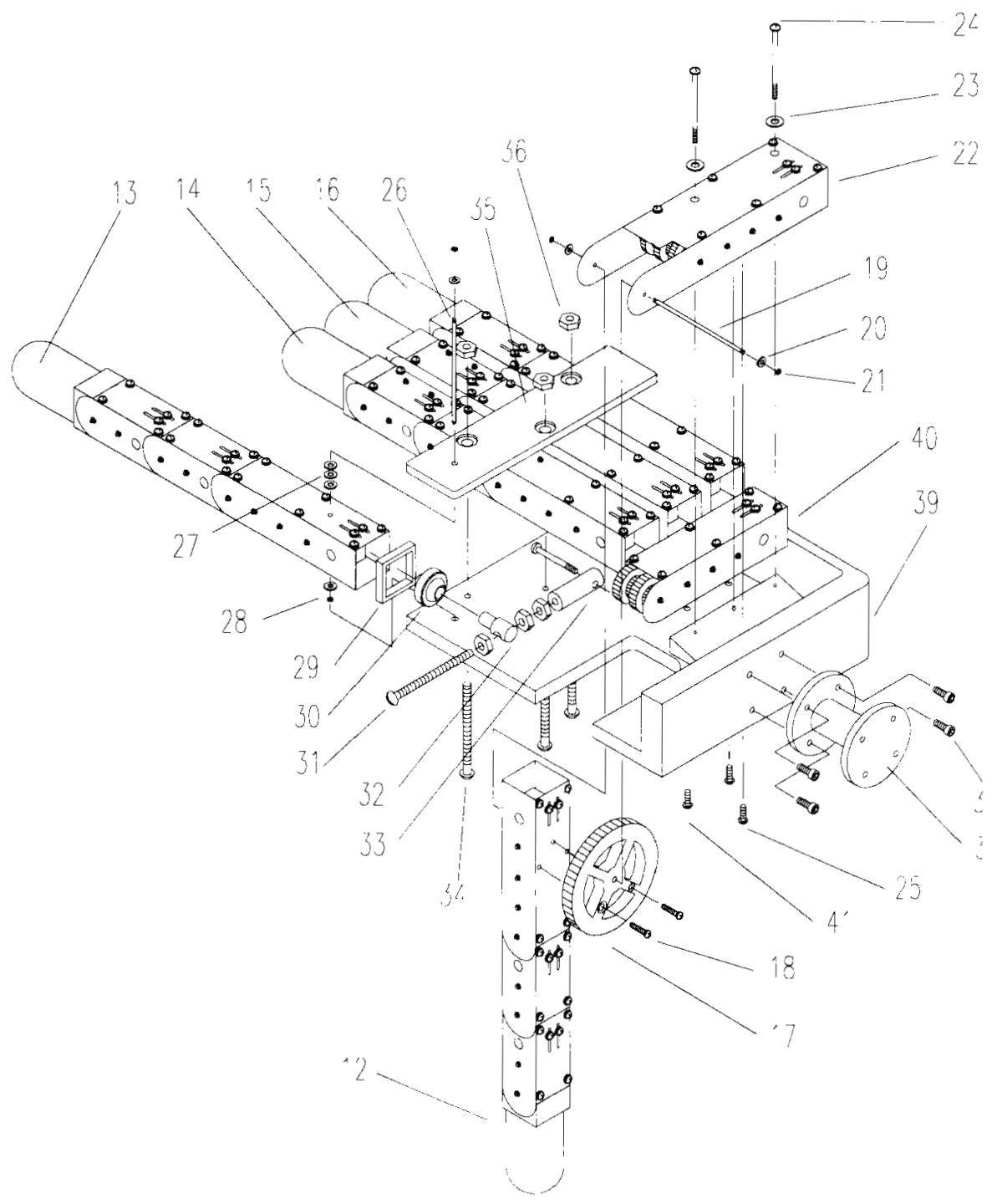

FIG. 5. The assembly of the NTU-Hand (parts 1-12 are listed in Fig. 3) is shown.

the addition of a glove of artificial skin, can be used for prosthetic applications.

\section{The required space}

As a result of the mechanism of the NTU-Hand, the mechanical parts are all packed inside the hand itself, and the size of the hand is very compact. However, the essential requirements of the artificial hand are not just the mechanism. They include electronic parts, such as controller, interface, power supply, and so on. The specially designed controller is also built to satisfy the space limitation $(10,12)$. Once the size of the controller is compact, the controller boards can mount on the back of the NTUHand (shown at the top of Fig. 5).

It is clear that the mechanical and electronic parts are integrated into the NTU-Hand itself and occupy a small space. This also explains why the NTU-Hand is suitable for prosthetic application.

\section{Effort of the fabrication and maintenance}

As shown in Figs. 3 and 5, most of the same parts in the finger segments are repeatedly used. The modular design both lowers the fabrication cost and eases the maintenance efforts.

\section{The potential of improvement}

The most efficient way to improve the performance of the hand is to change the materials of the hand. The selection of the material depends on the application of the hand. The prototype of the NTU-Hand, as shown in Fig. 1, is built of steel and aluminum except for the polymer fingertips. The weight of the hand, which is appropriate for robotics applications, is listed in Table 2. Because of the compact size of the whole system, the high possibility of prosthetic applications is one advantage of the NTU-Hand. For prosthetic applications, the material can be chosen to reduce the weight without 


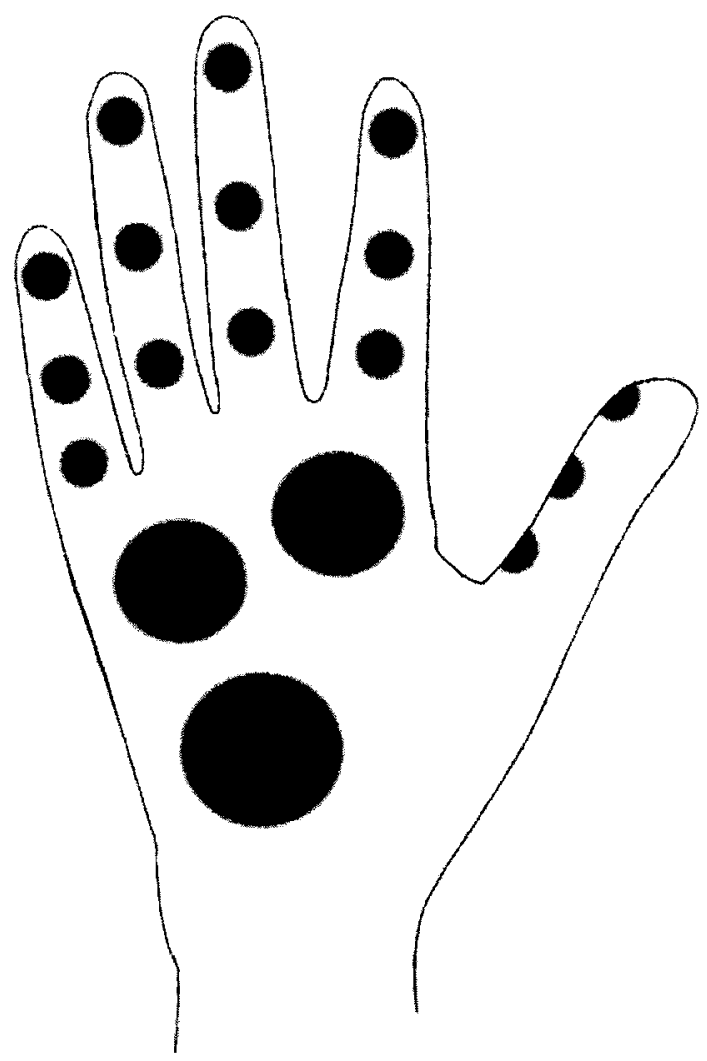

FIG. 6. Locations of 18 sensor pads are shown.

decreasing the strength; for instance, some polymer or composite material may be selected. The material of the hand can be chosen in terms of different applications, but the design spirit of the hand either will not change or will be just slightly modified.

\section{Adaptation for control and prosthetics}

Because of the mechanism configuration of the NTUHand, the control of the finger can be treated as a linkage robot arm. It allows the use of the analysis of dynamics and control techniques in the robotics. Hence, the control of the NTU-Hand can be treated as a problem of control and sensor integration with finger joint and tactility. To this end, the control system of NTU-Hand can be implemented compactly to embed the hand itself.

Because the NTU-Hand is compact, it has potential for prosthetic use. Choosing the material of the NTU-Hand is the first consideration. However, there are other applications for the NTU-Hand in prosthetics. One important work is obtaining the myoelectric signal of human and performing recognition (13-15). A cosmetic glove is also needed when the NTU-Hand is used in rehabilitation. These uses require further development. The power supply of the NTU-Hand may be replaced by a battery. The type of battery and its corresponding properties are also research topics to be pursued to use the NTU-Hand in prosthetics.

\section{Problems of the present design}

The current design suffers from some problems. These problems determine the performance of the NTU-Hand. This section states the problems encountered in the present design and shows how to solve them.

\section{Problems of the gear driver}

The driver mechanism of the NTU-Hand is performed by a set of gear trains. The disadvantage of the gear driver is the backlash problem, which means that the accuracy of the joint position will not be high. The problem is not serious when the hand is being operated to grasp an object. The backlash will not happen because the gears are maintaining contact during the grasp operation. The most important thing is tactile sensing while grasping.

The sensing of the NTU-Hand is divided into two parts: joint position sensing and force tactile sensing. According to the grasp of the human, the posture of the hand is not very accurate because the main goal of grasping is to manipulate an object. This means the function of the finger movement maintains contact with the object. The design of the NTU-Hand follows the guideline that tactile sensing is the first consideration and the movement of the finger joint is the second. It also implies that the cost in the hand design can be reduced.

\section{Force, torque, and heat sink}

The torque output of the finger segment is high because of the high gear ratio. Hence, the force of the hand will be large. The limitation of the force output is determined by the capacity of the motor and the strength of the gears. The modulus and thickness of the final stage of the gear trains should be larger than those of the previous

TABLE 1. Typical specifications of FSR (II)

\begin{tabular}{|c|c|c|}
\hline Parameter & Value & Conditions \\
\hline Force sensitivity range & $30 \mathrm{~g}-10 \mathrm{~kg}$ & \\
\hline Part-to-part force & $\pm 15 \%$ full scale & Typical part with consistent actuation \\
\hline Single part force & $\pm 2 \%$ full scale & \\
\hline Force resolution & Better than $0.5 \%$ full scale & \\
\hline Device rise time & 1-2 ms (mechanical) & \\
\hline Lifetime & $>10$ million actuation & \\
\hline Use temperature & $-30^{\circ}-+170^{\circ} \mathrm{C}$ & High temperature adhesives \\
\hline
\end{tabular}




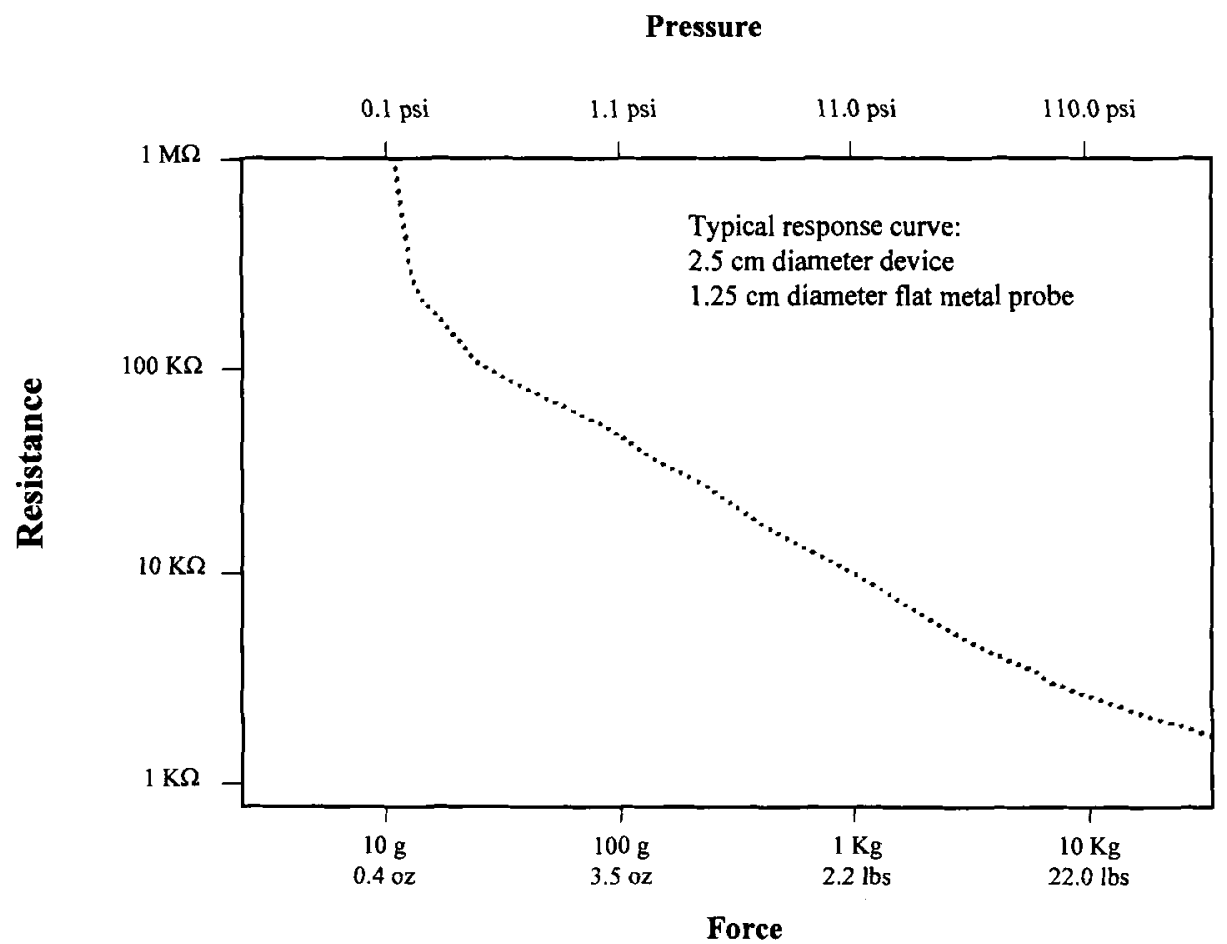

FIG. 7. FSR force/resistance characteristics (11) are given.

stage to stand the larger torque. Alternatively, a high strength gear may be used in the final stage of the gear trains.

The heat sink of the motor is also related to the force output. The torque of the motor decreases while the heat accumulates in the chamber of the finger segment. In the prototype of the NTU-Hand, the seat of the motor (part 9 of Fig. 3) is used to transfer the heat to the outside of the finger segment.

\section{Material and applications}

The selection of the material depends on the intended application of the hand. The prototype of the NTU-Hand, as shown in Fig. 1, is built almost entirely of steel and aluminum except for the polymer fingertip. The weight of the hand, which is appropriate for the robotics appli- cation, is listed in Table 2. For prosthetic applications, the material can be chosen to reduce the weight without decreasing the strength. Moreover, the design spirit of the hand either will not change or will be just slightly modified.

\section{Specification of the prototype}

Because the fundamental finger of the NTU-Hand has 3 joints numbered from 1 to 3 , the specification of the 3 joints of each finger are all the same. The lateral joints (joint 0 ) of the thumb and the first finger are performed by different auxiliary devices. The specifications of these 2 joints are special.

The specifications of the NTU-Hand are listed in Table 2. The prototype of the NTU-Hand is made of metal. Its weight can be reduced when other materials are

TABLE 2. The specifications of prototype of the NTU-Hand

\begin{tabular}{|c|c|c|c|c|c|}
\hline & $\begin{array}{l}\text { Joint } 0 \text { of } \\
\text { the thumb }\end{array}$ & $\begin{array}{l}\text { Joint } 0 \text { of } \\
\text { the first finger }\end{array}$ & $\begin{array}{l}\text { Joint } 1 \text { of } \\
\text { each finger }\end{array}$ & $\begin{array}{l}\text { Joint } 2 \text { of } \\
\text { each finger }\end{array}$ & $\begin{array}{l}\text { Joint } 3 \text { of } \\
\text { each finger }\end{array}$ \\
\hline Maximum angular velocity & $0.21 \mathrm{rad} / \mathrm{s}$ & $0.39 \mathrm{rad} / \mathrm{s}$ & $1.05 \mathrm{rad} / \mathrm{s}$ & $5.97 \mathrm{rad} / \mathrm{s}$ & $7.33 \mathrm{rad} / \mathrm{s}$ \\
\hline Bandwidth & $0.15 \mathrm{~Hz}$ & $0.31 \mathrm{~Hz}$ & $0.50 \mathrm{~Hz}$ & $2.85 \mathrm{~Hz}$ & $3.50 \mathrm{~Hz}$ \\
\hline Torque output & $3661.9 \mathrm{~g}-\mathrm{cm}$ & $1971.8 \mathrm{~g}-\mathrm{cm}$ & $1350.1 \mathrm{~g}-\mathrm{cm}$ & $859.4 \mathrm{~g}-\mathrm{cm}$ & $859.4 \mathrm{~g}-\mathrm{cm}$ \\
\hline \multicolumn{4}{|c|}{ The weight of each finger } & $191.2 \mathrm{~g}$ & \\
\hline \multicolumn{4}{|c|}{ The weight of the palm and auxiliary device } & 613.1 & \\
\hline \multicolumn{4}{|c|}{ The total weight of the NTU-Hand } & $1,569.0 \mathrm{~g}$ & \\
\hline \multicolumn{4}{|c|}{ The rated weight of the object to be manipulated } & 0.51 & \\
\hline \multicolumn{4}{|c|}{ The rated weight of the object to be grasped } & 1.01 & \\
\hline \multicolumn{4}{|c|}{ The maximum linear velocity of finger tip } & $877.08 \mathrm{r}$ & \\
\hline \multicolumn{3}{|c|}{ The power consumption of the whole hand mechanism } & & $0-29.8$ & \\
\hline
\end{tabular}


TABLE 3. The link parameters of finger

\begin{tabular}{lccccc}
\hline & $\begin{array}{c}\theta_{j} \\
\text { (degree) }\end{array}$ & $\begin{array}{c}\alpha_{j} \\
(\text { degree })\end{array}$ & $\begin{array}{c}a_{j} \\
(\mathrm{~mm})\end{array}$ & $\begin{array}{c}d_{j} \\
(\mathrm{~mm})\end{array}$ & $\begin{array}{c}\text { Joint range } \\
\text { (degree) }\end{array}$ \\
\hline Joint 0 $0^{a, b}$ & 0 & 90 & $35.2^{a, b}$ & 0 & $-30-45^{a}$ \\
Joint 1 & 0 & 0 & 34.0 & 0 & $-10-10^{\mathrm{b}}$ \\
Joint 2 & 0 & 0 & 34.0 & 0 & $0-60$ \\
Joint 3 & 0 & 0 & 42.0 & 0 & $0-60$ \\
\hline
\end{tabular}

${ }^{a}$ Thumb
${ }^{b}$ First finger

used. The rated weight of the object to be manipulated dynamically is determined by dexterous operation while the object is operated by the fingertips. The rated weight of object to be grasped statically is determined by power grasp operation while the object is grasped by the fingertip, the inner links of the finger, and the palm of the hand.

\section{THE WORKSPACE OF THE NTU-HAND}

If each finger is treated as an individual unit, the fingers are similar to the chained linkages. Note that the thumb and the first finger have 4 joints numbered from 0 to 3 , and the other 3 fingers only have 3 joints numbered from 1 to 3 . The link parameters of each finger are listed in Table 3, based on the Denavit-Hartenberg method (16).

The dexterous workspace of one finger is shown in Fig. 8 , which is obtained by direct kinematics. Notice that the density of marks shows the dexterity of the fingertip. The thumb and the first finger have more dexterity for the sake of the lateral joint. They expand the plane workspace into a three-dimensional space. If each contact point lies in the workspace and a corresponding inverse kinematics can be found, the artificial hand can perform a dexterous manipulation.

\section{COMPUTER SIMULATIONS}

Because it is difficult to numerically interpret the posture of the multifingered hand with 17 joint variables and the relationship with the grasped object, a threedimensional graph interface is designed for the simulation, as shown in Fig. 9. The simulation is performed on a SUN sparc 10 workstation under the X environment. The user can define the positions and orientations of the object, and the hand can then set the grasp posture of the NTU-Hand. The relationship between the hand and the object can be viewed from any angles.

After the relationship between the hand and the object is determined, the corresponding posture can be shown in our simulation. The first case is a disc object, as shown in Fig. 10. The cases of rectangular and cylindrical objects are also shown in Figs. 11 and 12. The computer

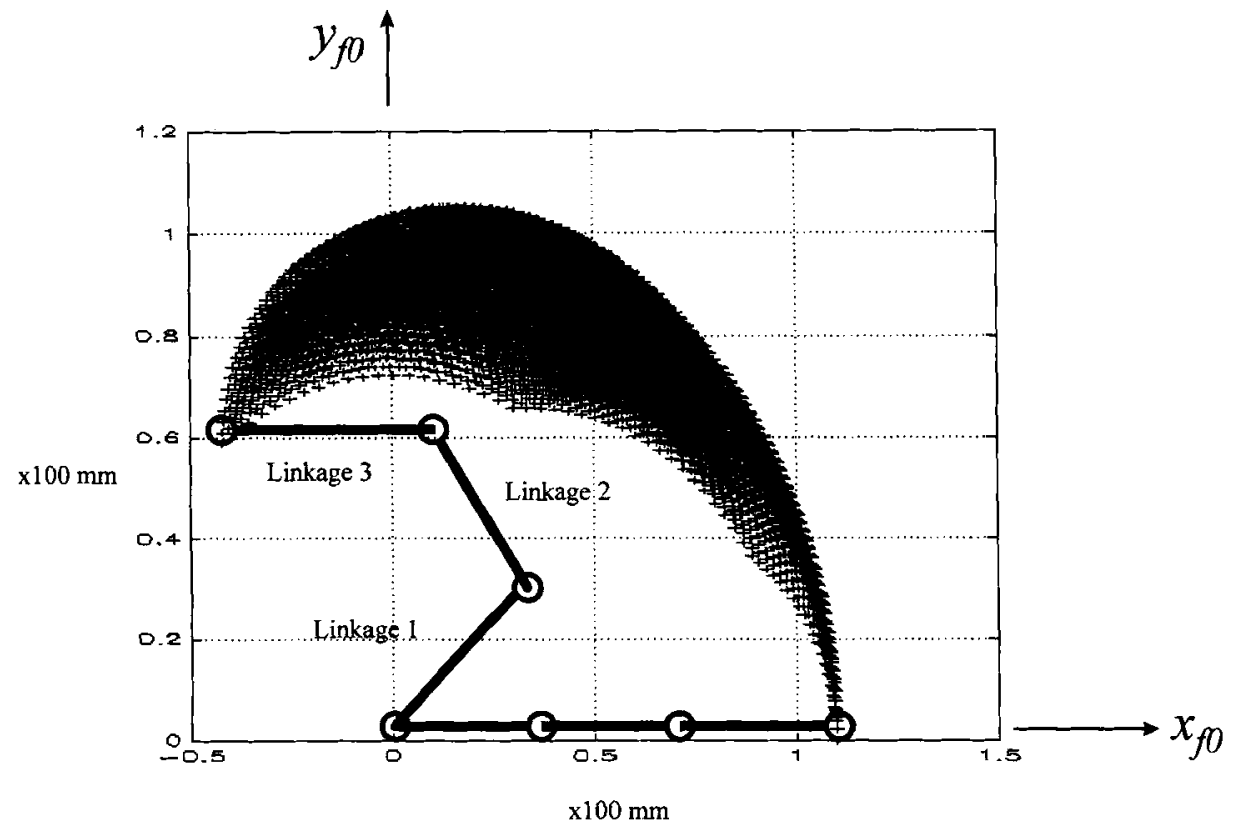

FIG. 8. The workspace of the finger is shown. 


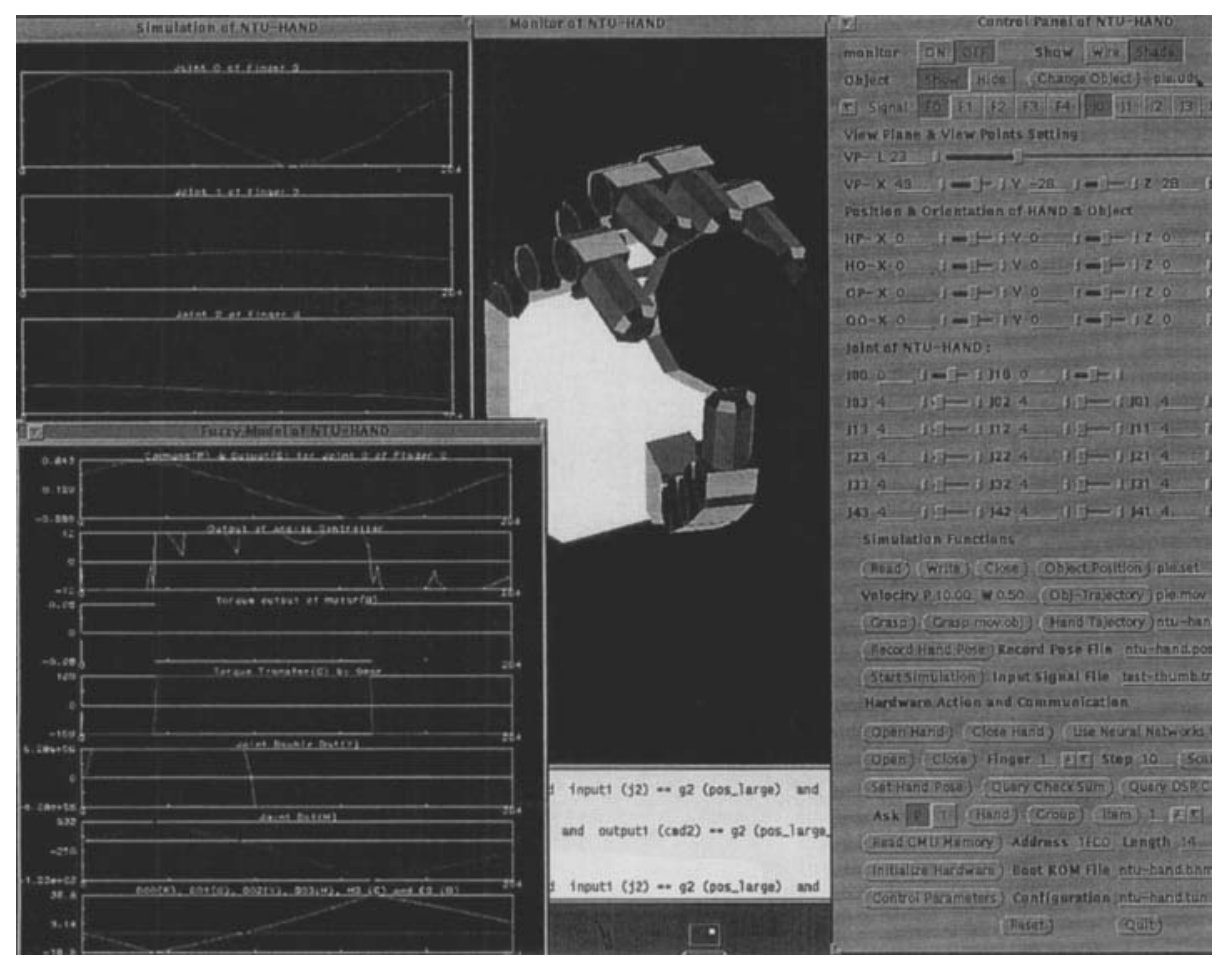

FIG. 9. The 3-dimensional user interface is pictured.

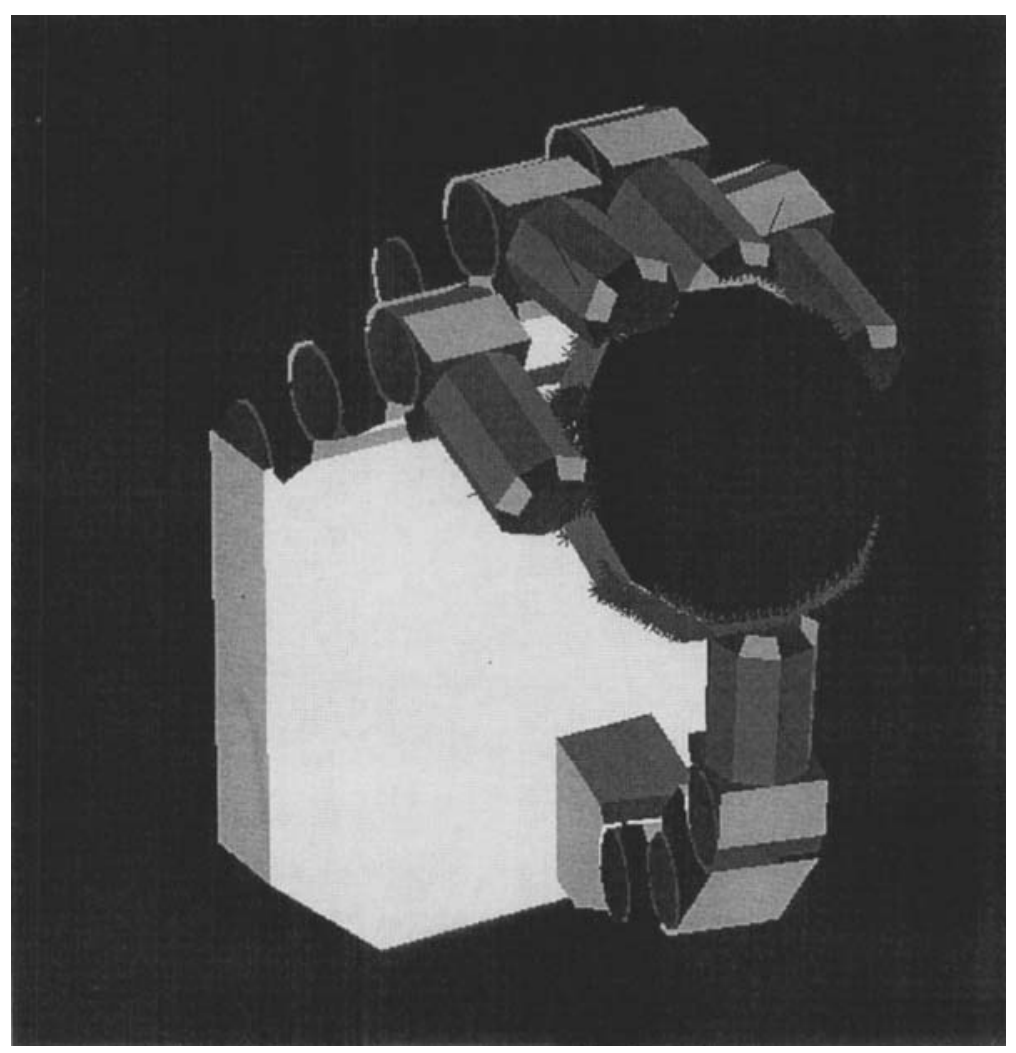

FIG. 10. The postures for grasping disc object are shown. 


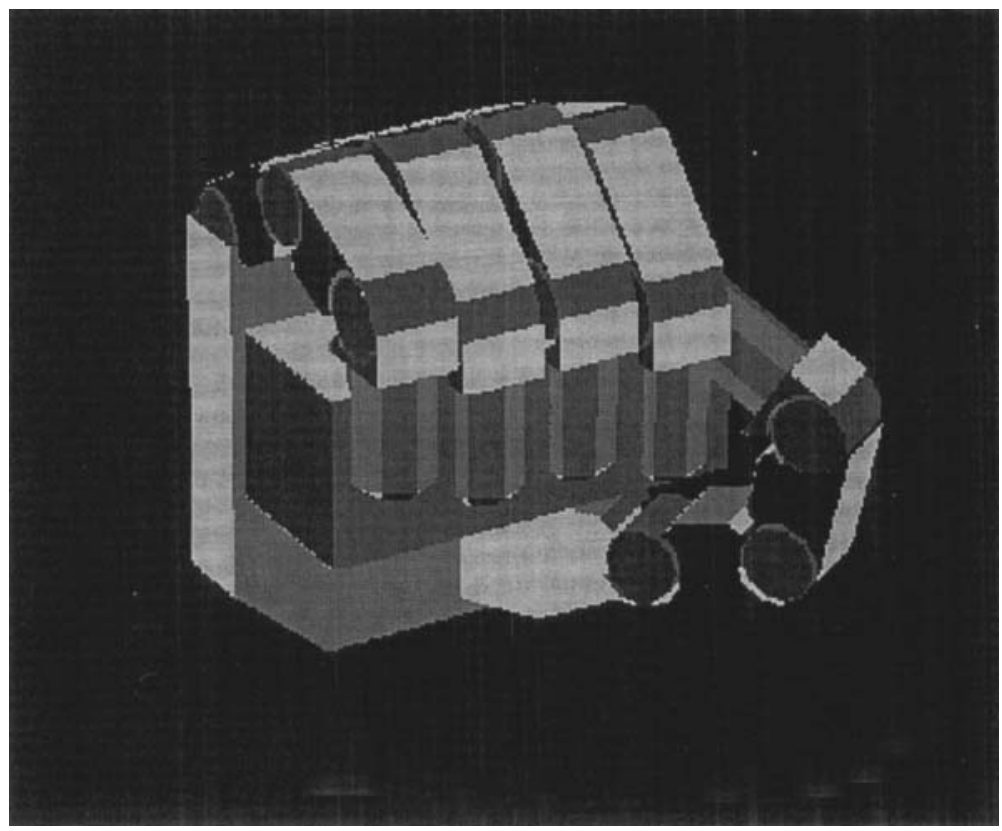

FIG. 11. The postures for grasping rectangular objects are shown.

simulation shows the grasp posture and capacity of the NTU-Hand to enable us to examine the mechanism design.

\section{CONCLUSIONS}

The dexterous artificial hand NTU-Hand, with its 17 DOFs, has been designed and fabricated to achieve dex- terous manipulation. Because the NTU-Hand is lighter and much more compact than traditional fingered hands, and because it has an uncoupled driven mechanism, it can be easily attached to the end of a robot arm to perform dexterous manipulation. In particular, it is useful for prosthetic applications. According to the mechanical structure of the NTU-Hand, the specifications, workspace, and computer simulation results to show grasp ability are also presented in this paper.

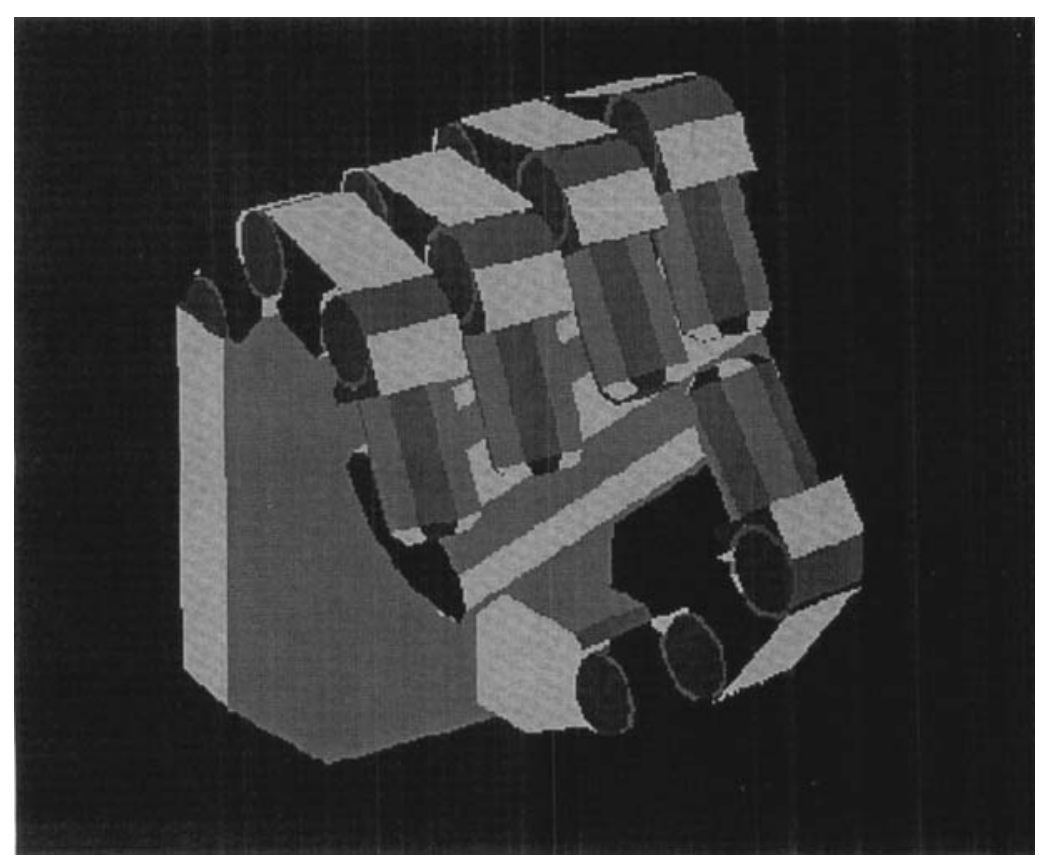

FIG. 12. The postures for grasping cylinder objects are shown. 


\section{REFERENCES}

1. Jacobsen SC, Wood JE, Knutti DF, Biggers KB. The UTAH/MIT dexterous hand: Work in progress. Int J Robotic's Res 1984:3.4: $21-50$.

2. Speeter TH. Primitive based control of the Utah/MIT dexterous hand. IEEE Int Conf Robotics Automat 1991:868-77.

3. Tomovic R, Bekey GA, Karplus WJ. A strategy for grasp synthesis with multifingered robot hand. Int Conf Robotics Automat 1987: 83-9.

4. Umetsu M, Oniki K. Compliant motion control of arm-hand system. JSME Int Conf Adv Mechatronics 1993:429-32.

5. Stansfield SA. Knowledge-based robotic grasping. IEEE Conf Robotics Automat 1990:1270-5.

6. Speeter TH. Control of the Utah/MIT dexterous hand: Hardware and software hierarchy. J Robotics Syst 1990;7(5):759-90.

7. Lin LR. Development of a dexterous hand: NTU-Hand. Ph.D. Thesis, Department of Mechanical Engineering, National Taiwan University, R.O.C.. 1996.

8. Mirtich B, Canny J. Easily computable optimum grasps in 2-D and 3-D. IEEE Int Conf Robotics Automat 1994:739-47.
9. Fowler I. Human anatomy. Belmont. CA: Wadsworth Publishing, 1984.

10. Huang HP, Lin LR. Development of a prosthetic hand with multifingers. NSC Rep R.O.C. NSC 83-0422-E002-093, 1994.

11. Interlink Electronics Inc. FSR technical specifications. CA: 1989.

12. Lin LR. Huang HP. DSP-based fuzzy control of a multifingered robot hand. IEEE Int Conf Systems, Man Cybernet 1995:3672-7.

13. Gibbsons DT, O'Riain MD, Philippe-Auguste S. An above-elbow prosthesis employing programmed linkages. IEEE Trans Biomed Eng 1987:7:493-8.

14. Graupe DG, Magnussen J, Beex AA. A microprocessor system for multifunctional control of upper-limb prostheses via myoelectric signal identifications. IEEE Trans Auto Contr 1978;4:538-44.

15. Myers DR, Moskowitz GD. Myoelectric pattern recognition for use in the volitional control of above-knee prostheses. IEEE Trans Systems, Man Cybernet 1981;4:296-302.

16. Denavit J, Hartenberg RS. A kinematics notation for low-pair mechanism based on matrices. $J$ Appl Mech 1955:2:215-21. 